\title{
Contamination des escargots prélevés dans la vallée de l'Ouémé au Bénin par des métaux lourds et évaluation des risques sanitaires
}

\author{
Mahoudjro Roméo ADAMOU ${ }^{1 *}$, Elisabeth YEHOUENOU AZEHOUN PAZOU ${ }^{1}$ et \\ Adjouavi Yvette DEGUENON ${ }^{2}$
}

${ }^{1}$ Laboratoire de Recherche en Biologie Appliquée (LARBA), Département de Génie de l'Environnement, Ecole Polytechnique d'Abomey-Calavi (EPAC), Université d'Abomey-Calavi (UAC), 01 BP 526, Cotonou, Bénin.

${ }^{2}$ Laboratoire de Recherche en Biochimie et Toxicologie de l'Environnement (LaRBiTE), Département de Biochimie et de Biologie Cellulaire, Faculté des Sciences et Techniques (FAST), Université d'Abomey-Calavi (UAC), 03 BP 0994 Cotonou, Bénin.

*Auteur correspondant ; E-mail: adamour87@gmail.com; Tél.: +22997379803

\section{RESUME}

Les escargots de la famille des Achatinidae, très appréciés par les populations béninoises, sont des espèces qui ont la capacité d'absorber et de concentrer dans leur organisme certaines substances chimiques toxiques comme les métaux lourds. La présente étude a pour objectif de déterminer la concentration des métaux lourds $(\mathrm{Pb}$ et $\mathrm{Cd})$ dans deux espèces d'escargots bouillis et frits de la vallée de l'Ouémé et d'évaluer les risques sanitaires encourus par les populations. La méthode d'analyse utilisée pour le dosage des métaux lourds dans les échantillons des espèces Archachatina marginata et Limicolaria spp collectés est la voltampérométrie inverse. Il ressort des résultats que dans les escargots bouillis, les concentrations moyennes du cadmium et du plomb varient respectivement entre 0 et $0,046 \mathrm{mg} / \mathrm{kg}$ puis entre 0,035 et $0,096 \mathrm{mg} / \mathrm{kg}$ pour Archachatina marginata et entre 0 et $0,422 \mathrm{mg} / \mathrm{kg}$ puis entre 0,016 et $1,024 \mathrm{mg} / \mathrm{kg}$ pour Limicolaria spp. Dans les escargots frits, les concentrations moyennes du cadmium et du plomb varient respectivement entre 0,003 et $0,067 \mathrm{mg} / \mathrm{kg}$ puis entre 0,023 et $0,062 \mathrm{mg} / \mathrm{kg}$ pour l'Archachatina marginata et entre 0 et $0,769 \mathrm{mg} / \mathrm{kg}$ puis entre 0 et 0,275 $\mathrm{mg} / \mathrm{kg}$ pour Limicolaria spp. L'évaluation des risques sanitaires a révélé que le quotient de danger du cadmium est supérieur à 1 pour les adultes et les enfants dans les deux espèces d'escargots bouillis et frits dans plusieurs localités. La consommation des escargots provenant de ces localités présenterait donc un danger pour la santé des adultes et encore plus des enfants à cause de l'accumulation du cadmium dans la viande des escargots malgré les méthodes de cuisson. Il urge donc que les populations réduisent la quantité d'escargots consommés par repas ainsi que la fréquence.

(C) 2019 International Formulae Group. All rights reserved.

Mots clés: Escargots, métaux lourds, risques sanitaires, vallée de l'Ouémé.

\section{Contamination of snails collected in the Ouémé valley in Benin by heavy metals and health risk assessment}

\begin{abstract}
Snails meat of the Achatinidae family, highly appreciated by Beninese, are species that have the capacity to absorb and concentrate in their body some toxic chemicals such as heavy metals. The present study
\end{abstract}


aims to determine heavy metals concentration $(\mathrm{Pb}$ and $\mathrm{Cd}$ ) in two species of boiled and fried snails of the Ouémé valley and to evaluate the health risks to the populations. Analytical method used for the determination of heavy metals in samples of the species Archachatina marginata and Limicolaria spp collected is reverse voltammetry. Results showed that in the boiled snails, the mean concentrations of cadmium and lead ranged from 0 to $0.046 \mathrm{mg} / \mathrm{kg}$ and from 0.035 to $0.096 \mathrm{mg} / \mathrm{kg}$ for Archachatina marginata and from 0 to $0.422 \mathrm{mg} / \mathrm{kg}$ and from 0.016 to 0.022 . $1.024 \mathrm{mg} / \mathrm{kg}$ for Limicolaria spp. In fried snails, the mean concentrations of cadmium and lead range from 0.003 to $0.067 \mathrm{mg} / \mathrm{kg}$ and from 0.023 to $0.062 \mathrm{mg} / \mathrm{kg}$ for Archachatina marginata and from 0 to $0.769 \mathrm{mg} / \mathrm{kg}$ and from 0 to $0.275 \mathrm{mg} / \mathrm{kg}$ for Limicolaria spp. Health risk assessment revealed that the hazard quotient for cadmium is greater than 1 for adults and children in both boiled and fried snail species in many localities. Consumption of snails from these localities would therefore pose a danger to adults health and even more children because of cadmium accumulation in snail meat despite the cooking methods. It is therefore urgent that populations reduce snails amount consumed per meal and the frequency.

(C) 2019 International Formulae Group. All rights reserved.

Keywords: Snails, heavy metals, health risks, Ouémé Valley.

\section{INTRODUCTION}

Il est indéniable de nos jours que, dans les pays en développement, les ressources alimentaires non conventionnelles (les espèces animales et végétales qu'on trouve à l'état naturel et qui sont consommées par l'homme ou les animaux) jouent un rôle assez substantiel dans l'alimentation des populations (Sodjinou et al., 2002). Au nombre de ces ressources, on retrouve notamment des escargots géants africains (ou achatines). Ces escargots appartiennent à la famille des Achatinidae dont la chair est très appréciée par de nombreuses populations africaines (Sika Piba et al., 2014 ; Bouye et al., 2017), et celles béninoises en particulier. En effet, le céphalopode (la partie consommée de l'escargot) représente, d'après une étude réalisée en Côte d'Ivoire par Otchoumou (2005), 30\% du poids vif de l'escargot. Il est très riche en eau (80\%) et renferme en matière sèche 62 à $75 \%$ de protéines brutes. Bien que pauvre en matière grasse, il possède une valeur calorifique très intéressante (341 à 358 $\mathrm{Kcal} / 100 \mathrm{~g})$, une teneur élevée en calcium $(1,3$ $\mathrm{g} / 100 \mathrm{~g}$ MS) et en fer (491 mg/Kg MS). Presque tous les acides aminés essentiels dont l'homme a besoin y sont représentés avec une teneur en lysine allant jusqu'à 4,5 g/16 g N. Les vitamines $\mathrm{A}, \mathrm{D}_{3}$ et $\mathrm{E}$ sont également présentes. La chair des escargots représenterait la «viande de brousse» la plus prisée et la plus consommée en Côte d'Ivoire, après l'aulacode, avec près de 17.000 tonnes par an (Mbétid-Bessane, 2006). Au Bénin, leur consommation était estimée à 300 tonnes par an (Mbétid-Bessane, 2006). D'après Sodjinou et al. (2002), la viande d'achatine est la viande la plus consommée au Sud-Bénin devant l'aulacode, le poulet, l'ovin ou le caprin, le bœuf et le porc.

Les escargots sont des espèces bioaccumulatrices, capables d'absorber et de concentrer dans leur organisme certaines substances chimiques toxiques comme les métaux lourds. Ainsi, la pollution du biotope de ces escargots fait planer un risque de bioaccumulation des polluants chimiques. $\mathrm{Vu}$ que les escargots occupent une place de choix dans les assiettes des béninois, alors que les métaux lourds, quasi-totalement indestructibles dans l'environnement (Miquel, 2001), sont capables de pénétrer directement dans la chaîne alimentaire et se concentrer dans certains organismes vivants, exposant l'Homme à une intoxication lente mais certaine, la contamination des escargots constituerait un véritable problème de santé publique. Si plusieurs études ont montré la contamination des poissons par les métaux lourds (Yehouenou et al., 2006a et 2006b ; Youssao et al., 2011a et 2011b ; Hounkpatin et al., 2012), très peu se sont intéressées à la contamination des escargots. Ainsi, la présente étude se propose de déterminer la concentration des métaux lourds $(\mathrm{Pb}$ et $\mathrm{Cd}$ ) dans les espèces d'escargots cuits (bouillis et frits) de la vallée de l'Ouémé et d'évaluer les risques sanitaires encourus par les populations. 


\section{MATERIEL ET METHODES}

\section{Zone d'étude}

La présente étude a été réalisée dans la vallée de l'Ouémé, plus précisément dans la basse vallée de l'Ouémé comprise entre les parallèles $7^{\circ} 12^{\prime}$ et $6^{\circ} 23^{\prime}$ Nord et les méridiens $2^{\circ} 21^{\prime}$ et $2^{\circ} 33^{\prime}$ Est (Sossou-Agbo, 2013). Localisée dans la région Sud-Est du Bénin, elle est une plaine d'inondation en forme d'un triangle allongé mesurant $90 \mathrm{~km}$ du Nord au Sud (Figure 1). Huit (08) stations ont été retenues à travers les quatre communes de la zone d'étude. Il s'agit des localités de Kessounou, de Hozin, d'Akpadanou, de Todé, d'Affamè, d'Agonhoui, de Bembè et de Houinta.

\section{Matériel animal}

Cette étude a porté sur deux espèces d'escargot appartenant à la famille des Achatinidae. Il s'agit de Archachatina marginata et Limicolaria spp. Ce sont des espèces très prisées dans le Sud du Bénin et particulièrement dans les départements de l'Ouémé et du Plateau. Elles sont consommées par les populations sous forme bouillie et frite.

\section{Echantillonnage et prélèvements des escargots}

Quarante (40) individus d'escargots ont été prélevés par espèce directement auprès des ramasseurs dans toutes les localités retenues. Ils ont été immédiatement mis dans les bocaux en plastique préalablement lavés et stérilisés à l'acide chlorhydrique. Les échantillons prélevés ont été gardés au froid (température, à préciser) jusqu'à leur acheminement au laboratoire. Au total, 32 échantillons ont été collectés sur les huit sites de prélèvements à raison de 4 échantillons par site.

\section{Préparation des échantillons d'escargots}

Une fois ramenés aux laboratoires, les escargots ont d'abord été mis à jeun pendant deux jours $(48 \mathrm{~h})$ afin d'éliminer la nourriture non absorbée et les fèces de leur tractus digestif. Ensuite plusieurs mesures ont été prises sur une quarantaine d'escargots de chaque site de prélèvement. Il s'agit du poids de l'escargot, de la longueur et du diamètre de sa coquille. Après des pools de 10 individus d'escargots chacun ont été constitués, mis dans des sachets stériles et conservés à $-80{ }^{\circ} \mathrm{C}$ jusqu'au moment des analyses. Le premier pool a servi aux analyses des escargots bouillis et le deuxième aux analyses des escargots frits. Puisque le dosage des métaux lourds porte sur le céphalopode c'est-à-dire le pied des escargots (la partie consommée par les populations), la dissection de chaque individu d'escargots a permis de séparer le pied des viscères, au niveau du bord du manteau, après leur décongélation et leur extraction des coquilles. Enfin, les escargots ont été découpés en petits morceaux dans chaque pool et mélangés ensemble afin de permettre une certaine homogénéisation des polluants dans le pool.

\section{Cuisson des escargots bouillis et frits}

Les pools d'escargots réservés pour être bouillis ont d'abord été lavés plusieurs fois à l'eau et à l'alun. L'alun est du sulfate double de potassium et d'aluminium hydraté, très utilisé par les populations locales pour non seulement enlever les saletés mais aussi la substance gluante de la chair des escargots. Ils ont été ensuite portés au feu dans de l'eau salée jusqu'à ébullition.

Quant aux escargots frits, ils ont en un premier temps subi les mêmes étapes décrites ci-dessus pour les escargots bouillis. Dans un second temps, ils ont été frits dans de l'huile chaude à environ $180{ }^{\circ} \mathrm{C}$ pendant 4 minutes.

\section{Minéralisation des échantillons et dosage des métaux lourds}

Cinq grammes d'escargot sont d'abord pesés par échantillon dans des creusets et ensuite séchés à l'étuve à $105{ }^{\circ} \mathrm{C}$ pendant 24 h. La minéralisation a été faite dans un four programmable dont la température a progressivement été portée à $550{ }^{\circ} \mathrm{C}$ jusqu'à l'obtention de la cendre pour faciliter l'extraction des métaux (Nica et al., 2012). La cendre a été recueillie dans des tubes Falcon qui ont aussi servi à la digestion de la cendre. 
Cette dernière a été dissoute dans $20 \mathrm{ml}$ de $\mathrm{HNO}_{3}(0,5 \mathrm{~N})$ et agitée jusqu'à l'obtention d'une solution transparente. L'échantillon digéré a été filtré à travers un papier filtre Whatman $\mathrm{N}^{\circ}$ 42. Enfin le volume de chaque échantillon a été porté à $50 \mathrm{ml}$ par un ajout de $30 \mathrm{ml}$ de $\mathrm{HNO}_{3}(0,5 \mathrm{~N})$ et gardé à température ambiante pour les analyses subséquentes.

La méthode d'analyse utilisée pour le dosage des métaux lourds est la voltampérométrie inverse. Les échantillons après minéralisation sont dilués à trente pour cent $(30 \%)$ avec de l'eau déionisée puis les métaux lourds ont été quantifiés grâce au Metalyser HM 3000 (Trace2O, Berkshire, Royaume-Uni). Les électrodes ont été placées et conditionnées en fonction du métal recherché. Le métal a été quantifié par le Metalyser dans $70 \mathrm{ml}$ du minéralisât auquel sont ajoutés les réactifs du kit correspondant au métal recherché (tampon et standard). A la fin de l'analyse, la concentration du métal est directement affichée sur une tablette connectée à la machine. Les concentrations réelles en cations métalliques dans le céphalopode des escargots sont déterminées selon l'équation suivante :

$$
\mathrm{C} \text { en ppm }(\mathrm{mg} / \mathrm{kg})=\frac{\mathrm{Cs}^{*} \mathrm{Vf}^{*} \mathrm{Fd}^{*} 10^{-3}}{\mathrm{P}}
$$

Tel que :

C : concentration finale ;

Cs : concentration de l'élément dans la solution ;

Vf : volume final de d'échantillon (50 ml);

Fd : facteur de dilution ;

$\mathbf{P}$ : Prise d'essai en $\mathrm{g}$ (poids frais) de l'échantillon.

Les métaux lourds, cadmium et plomb, ont été choisis dans cette étude car ils font partie des plus toxiques pour l'homme et sont plus fréquemment retrouvés dans l'environnement (Viala et Grimaldi, 2005).

\section{Méthode d'évaluation des risques sanitaires liés à la consommation des escargots contaminés par les métaux lourds}

L'évaluation des risques sanitaires liés à la consommation des escargots contaminés par les éléments traces métalliques a suivi trois principales étapes (Gay et al., 2007) : l'identification du danger, le choix des valeurs toxicologiques de référence (VTR) et l'évaluation de l'exposition de la population (Bisson et al., 2009).

Elle aboutit au calcul de la dose journalière d'exposition (DJE). Selon Bassey et al. (2014), il est plus pertinent de calculer la DJE en tenant compte des produits cuits que des produits crus. Ainsi, l'évaluation des risques sanitaires dans le cadre de cette étude a été faite à la base des concentrations de métaux lourds enregistrées dans les escargots bouillis et frits, tenant compte aussi du fait que les populations ne consomment pas les escargots sous leur forme crue. Les scénarios d'exposition où l'individu est le plus exposé (hypothèse maximaliste) ont été utilisés. En effet, par hypothèse, il a été considéré que la quantité journalière moyenne d'escargot ingérée par un enfant est la même que celle ingérée par un adulte. Cette quantité est déterminée par une enquête de terrain auprès de 202 ramasseurs d'escargots de la zone d'étude. L'enquête a révélé que les populations consomment en moyenne 8 escargots Archachatina marginata par repas 3 fois par semaine et 16 escargots Limicolaria spp par repas 2 fois par semaine.

La DJE des polluants liée à la consommation des escargots est déterminée comme suit :

\section{$\mathrm{DJE}=\mathbf{C} * \mathbf{Q} * \mathbf{F} / \mathbf{P}$}

Tel que :

DJE : Dose journalière d'exposition aux éléments traces $(\mathrm{mg} / \mathrm{kg} / \mathrm{j})$;

C: Concentration en éléments traces des escargots $(\mathrm{mg} / \mathrm{kg})$;

Q: Quantité d'escargots ingérés par jour $(\mathrm{kg} / \mathrm{j})$;

F : Fréquence d'exposition $(\mathrm{F}=1)$, il est sans unité ;

P : Poids corporel de la cible $(\mathrm{kg})$.

La quantité d'escargots ingérés par jour Q a été calculée en considérant que le céphalopode (la partie consommée de l'escargot) représente, d'après une étude réalisée en Côte d'Ivoire par Otchoumou (2005), 30\% du poids vif de l'escargot, et en 
tenant compte des 8 escargots Archachatina marginata et des 16 escargots Limicolaria spp consommés par repas puis de la mesure du poids des escargots au laboratoire. Le poids corporel moyen des enfants de 0 à 15 ans est de $28 \mathrm{~kg}$ et celui d'un adulte est conventionnellement égal à $70 \mathrm{~kg}$ selon l'Agence américaine de protection de l'environnement (US EPA) (ASTEE, 2003).

\section{Caractérisation du risque}

La caractérisation du risque pour les effets à seuil est exprimée par le quotient de danger (QD). Il est calculé pour la voie d'exposition orale de la manière suivante :

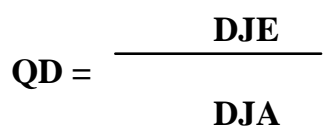

Tel que :

DJE : Dose Journalière d'Exposition $(\mathrm{mg} / \mathrm{kg} / \mathrm{j})$;

DJA : Dose Journalière Admise $(\mathrm{mg} / \mathrm{kg} / \mathrm{j})$.

Si QD $<1$, la survenue d'un effet toxique est très peu probable ;
Si QD > 1, l'apparition d'un effet toxique ne peut pas être exclue.

Les valeurs des doses journalières admises (DJA) les moins toxiques donc les plus faibles et les années de révision les plus récentes et issues des études épidémiologiques chez l'homme ont été privilégiées. Ainsi, selon le rapport de l'Institut National de l'Environnement Industriel et des Risques (INERIS) (Bisson et al., 2009), pour les effets chroniques à seuil, l'ATSDR (Agency for Toxic Substances and Disease Registry) recommande pour le $\mathrm{Cd} 2.10^{-4} \mathrm{mg} / \mathrm{kg} / \mathrm{j}$ et l'OMS préconise pour le $\mathrm{Pb} 3,6.10^{-3} \mathrm{mg} / \mathrm{kg} / \mathrm{j}$.

\section{Analyses statistiques}

Les données ont été soumises au test non paramétrique de Kruskall-Wallis de comparaison des moyennes et celui de Nruskal Kramer Nemenyi pour la structuration des moyennes au moyen du logiciel R 3.2.3.

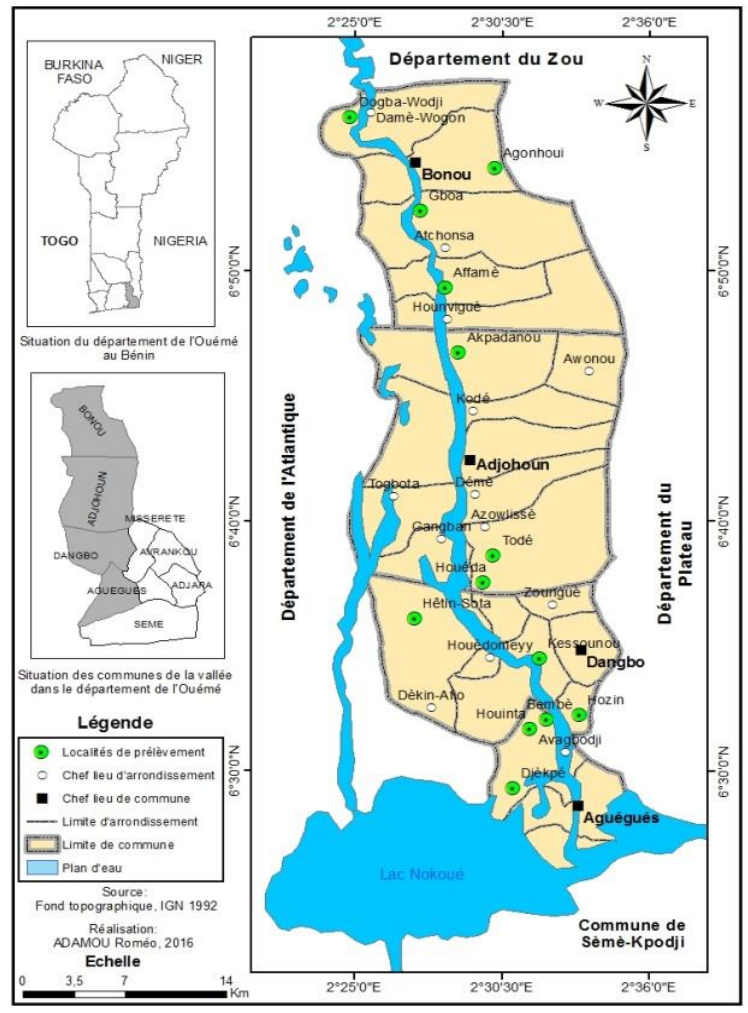

Figure 1: Localisation de la zone d'étude (vallée de l'Ouémé). 
RESULTATS

\section{Concentrations de métaux lourds dans les escargots bouillis}

Les résultats obtenus après l'analyse des échantillons d'escargots bouillis de la vallée de l'Ouémé ont révélé que pour Archachatina marginata, les concentrations moyennes du cadmium enregistrées varient entre 0 et $0,046 \mathrm{mg} / \mathrm{kg}$ tandis que celles du plomb varient entre 0,035 et $0,096 \mathrm{mg} / \mathrm{kg}$ (Figure 2). Par contre pour Limicolaria spp, les concentrations moyennes du cadmium enregistrées varient entre 0 et $0,422 \mathrm{mg} / \mathrm{kg}$ tandis que celles du plomb varient entre 0,016 et $1,024 \mathrm{mg} / \mathrm{kg}$ (Figure 3).

L'analyse des résultats après le test de Kruskall-Wallis pour la comparaison des moyennes et celui de Nruskal Kramer Nemenyi pour la structuration des moyennes a montré qu'il n'y a pas de différence significative $(P>0,05)$ entre les valeurs médianes des concentrations en métaux lourds (cadmium et plomb) contenues dans la viande bouillie des deux espèces d'escargot dans les différentes localités.

\section{Concentrations de métaux lourds dans les escargots frits}

Les résultats obtenus après l'analyse des échantillons d'escargots frits de la vallée de l'Ouémé ont révélé aussi que pour Archachatina marginata, les concentrations moyennes du cadmium enregistrées varient entre 0,003 et $0,067 \mathrm{mg} / \mathrm{kg}$ tandis que celles du plomb varient entre 0,023 et $0,062 \mathrm{mg} / \mathrm{kg}$ (Figure 4). Mais pour Limicolaria spp, les concentrations moyennes du cadmium enregistrées varient entre 0 et $0,769 \mathrm{mg} / \mathrm{kg}$ tandis que celles du plomb varient entre 0 et $0,275 \mathrm{mg} / \mathrm{kg}$ (Figure 5).

L'analyse des résultats après le test de Kruskall-Wallis pour la comparaison des moyennes et celui de Nruskal Kramer Nemenyi pour la structuration des moyennes a montré qu'il n'y a pas de différence significative $(P>0,05)$ entre les valeurs médianes des concentrations en métaux lourds (cadmium et plomb) contenues dans la viande frite des deux espèces d'escargot dans les différentes localités.
Risques sanitaires liés à la consommation des escargots contaminés aux métaux lourds

Les résultats de l'évaluation de l'exposition au $\mathrm{Cd}$ et au $\mathrm{Pb}$ liée à l'ingestion des escargots ainsi que les quotients de danger (QD) correspondants chez les adultes et les enfants sont présentés dans les tableaux 1 à 8 .

L'analyse des tableaux a révélé que pour le Cd dans les escargots Archachatina marginata frits, le quotient de danger est supérieur à 1 dans la localité d'Agonhoui chez les adultes $(\mathrm{QD}=2,21)$ et les enfants $(\mathrm{QD}=5,53)$, puis dans la localité d'Affamè chez les enfants seulement $(\mathrm{QD}=1,76)$. Toujours pour le $\mathrm{Cd}$ dans les escargots Archachatina marginata bouillis, on note aussi que le quotient de danger est supérieur à 1 dans la localité d'Agonhoui chez les adultes $(\mathrm{QD}=1,52)$ et les enfants $(\mathrm{QD}=3,80)$, puis dans la localité d'Affamè chez les enfants seulement $(\mathrm{QD}=2,22)$. Par contre pour le $\mathrm{Pb}$ dans ces escargots frits et bouillis, le quotient de danger est inférieur à 1 dans toutes les localités. On peut en déduire que la survenue d'un effet toxique provenant $\mathrm{du} \mathrm{Pb}$ et lié à la consommation d'escargots frits et bouillis de la vallée de l'Ouémé est très peu probable chez les adultes et les enfants. Il n'y a donc pas de risque d'effets toxiques lié au $\mathrm{Pb}$. Cependant l'on peut s'attendre à des effets toxiques provenant $\mathrm{du} \mathrm{Cd}$ et liés à la consommation d'escargots Archachatina marginata frits et bouillis à Agonhoui chez les adultes et les enfants, puis à Affamè chez les enfants. Notons que le risque est plus élevé chez les enfants en raison de leur faible poids corporel. Les localités d'Agonhoui et d'Affamè sont toutes dans la commune de Bonou. Ces résultats révèlent donc que la consommation des escargots Archachatina marginata provenant de la commune de Bonou présenterait un risque sanitaire pour les adultes et les enfants en raison de l'accumulation $\mathrm{du} \mathrm{Cd}$ dans la chair des escargots malgré la cuisson.

Quant aux escargots Limicolaria spp, l'analyse des tableaux révèle que pour le cadmium, le quotient de danger n'est supérieur à 1 que chez les enfants à 
Kessounou $(\mathrm{QD}=2,48)$ et à Todé $(\mathrm{QD}=1,33)$ pour la forme frite, puis à Todé $(\mathrm{QD}=1,23)$ et à Houinta $(\mathrm{QD}=2,08)$ pour la forme bouillie. Par contre pour le $\mathrm{Pb}$ dans ces escargots frits et bouillis, le quotient de danger est inférieur à 1 dans toutes les localités. On peut alors en déduire que la survenue d'un effet toxique provenant $\mathrm{du} \mathrm{Pb}$ et lié à la consommation d'escargots frits et bouillis de la vallée de l'Ouémé est très peu probable chez les adultes et les enfants. Il n'y a donc pas de risque d'effets toxiques lié au $\mathrm{Pb}$. Cependant l'on peut s'attendre à des effets toxiques provenant $\mathrm{du} \mathrm{Cd}$ et liés à la consommation d'escargots Limicolaria spp frits et bouillis à Kessounou, Todé et Houinta chez les enfants uniquement. Le risque élevé observé uniquement chez les enfants se justifie par leur faible poids corporel. Ces résultats révèlent donc que la consommation des escargots Limicolaria spp provenant de ces trois localités présenterait un risque sanitaire pour les enfants en raison de l'accumulation $\mathrm{du} \mathrm{Cd}$ dans la chair des escargots malgré la cuisson.

Les résultats du test $t$ de Student sur le logarithme népérien des valeurs individuelles de risque sanitaire diffèrent très significativement $(P \leq 0,01)$ entre les métaux lourds que ce soit chez les enfants ou chez les adultes (Tableau 9). Le risque sanitaire lié au cadmium est plus élevé (voire 10 fois) que celui de plomb et est plus élevé chez les enfants que chez les adultes. Aucune différence significative n'est observée pour les formes de cuisson.

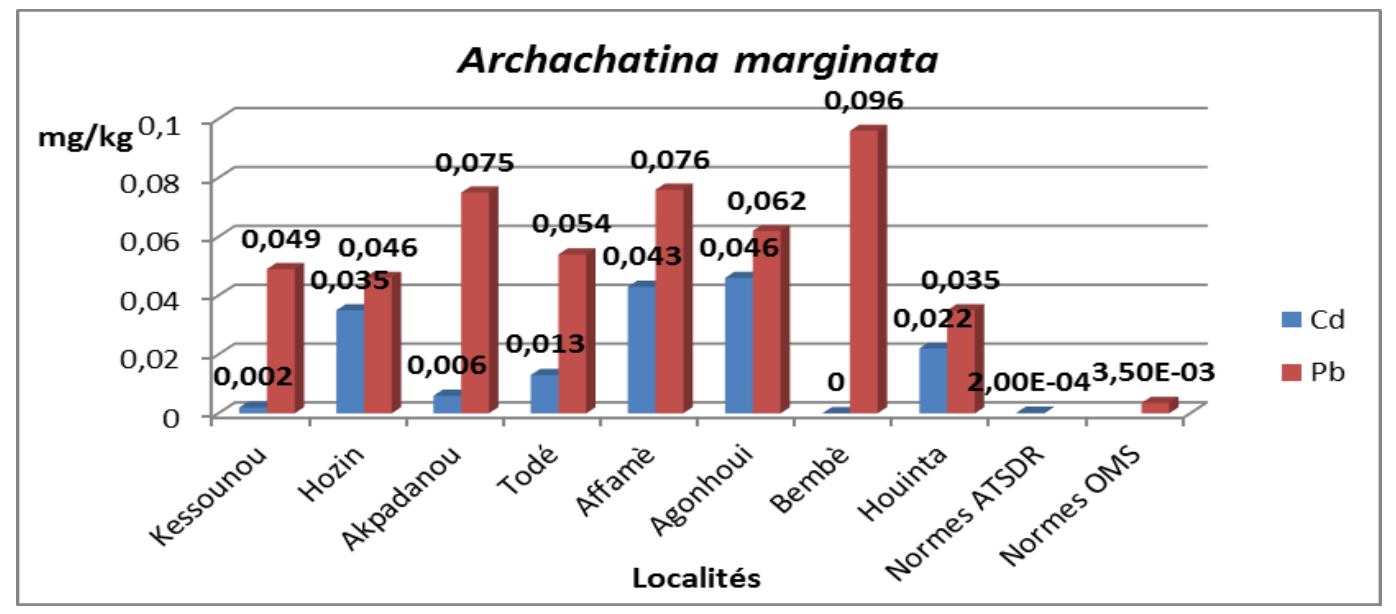

Figure 2: Concentrations de métaux lourds dans l'espèce Archachatina marginata bouilli.

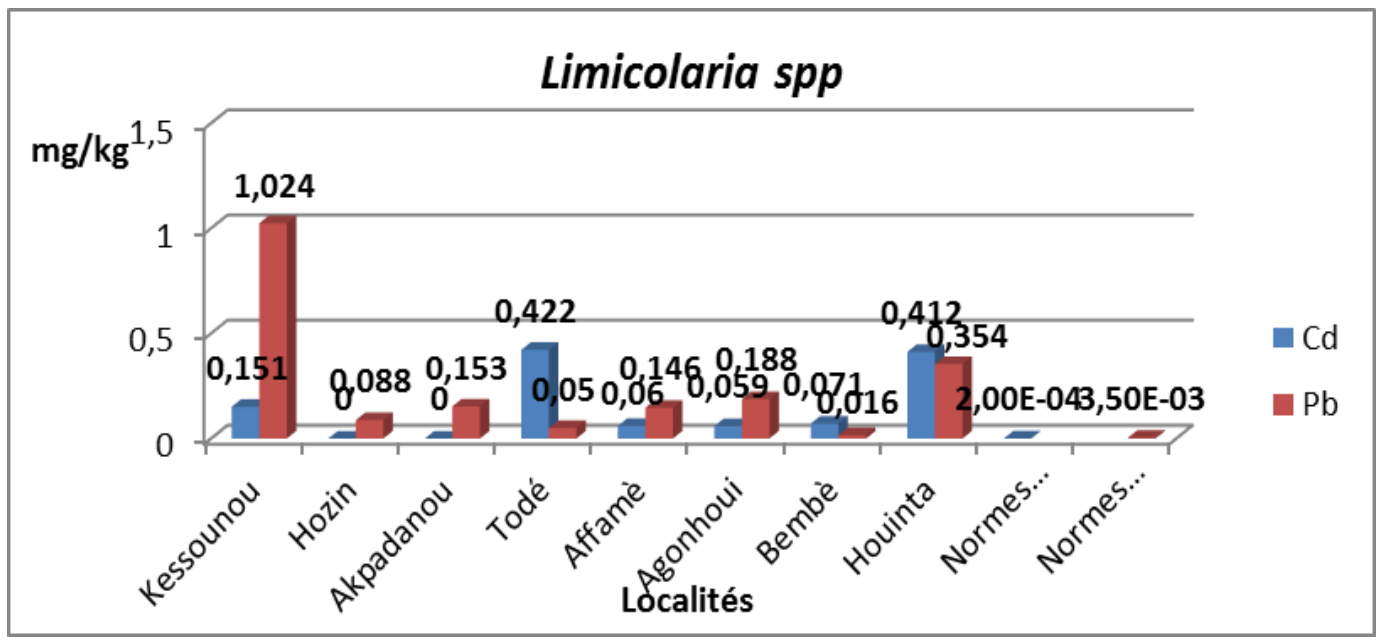

Figure 3 : Concentrations de métaux lourds dans l'espèce Limicolaria spp bouilli. 


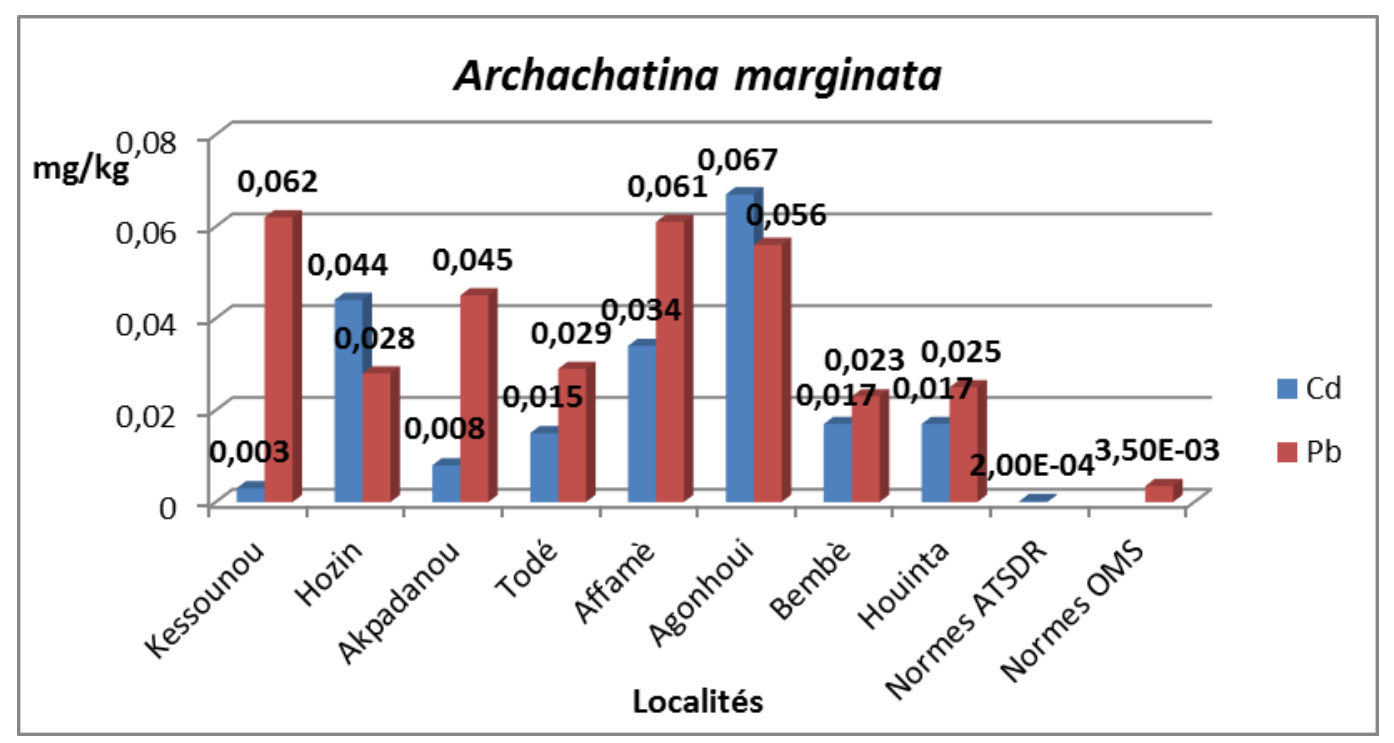

Figure 4 : Concentrations de métaux lourds dans l'espèce Archachatina marginata frit.

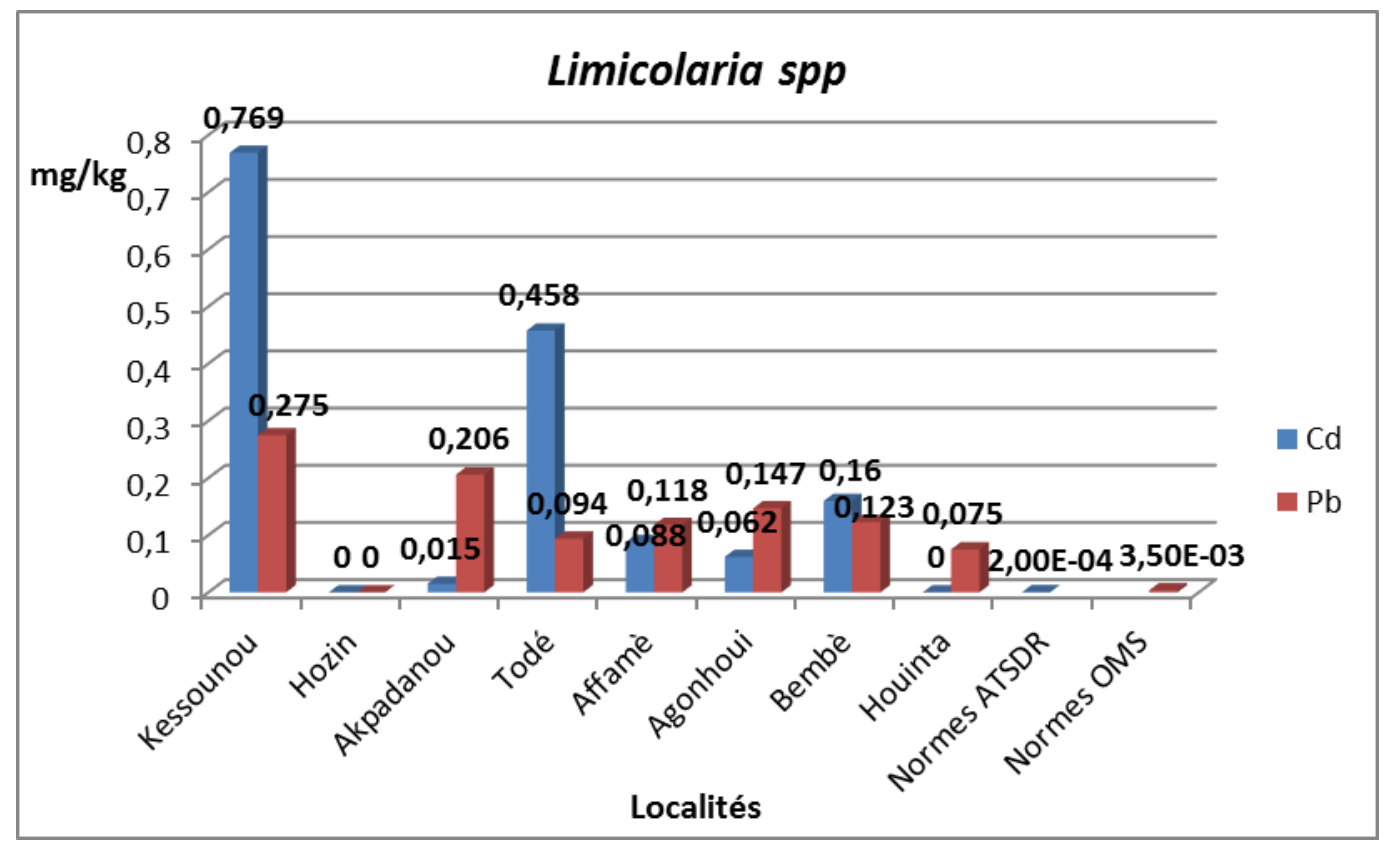

Figure 5 : Concentrations de métaux lourds dans l'espèce Limicolaria spp frit. 
Tableau 1: Risques sanitaires liés à la consommation d'escargots Archachatina marginata frits contaminés par le Cd dans la vallée de l'Ouémé.

\begin{tabular}{|c|c|c|c|c|c|c|c|c|c|c|}
\hline \multirow{2}{*}{ Cadmium } & \multirow{2}{*}{$\begin{array}{l}\text { Concentration } \\
\text { moyenne en métaux } \\
\text { lourds C (mg/Kg) }\end{array}$} & \multirow[b]{2}{*}{$\begin{array}{l}\text { Poids moyen des } \\
\text { escargots (kg) }\end{array}$} & \multirow{2}{*}{$\begin{array}{c}\text { Quantité } \\
\text { d'escargots } \\
\text { ingérés par jour Q } \\
(\mathrm{Kg} / \mathbf{j})\end{array}$} & \multicolumn{2}{|c|}{$\begin{array}{c}\text { Poids de la cible } P \\
(\mathrm{~kg})\end{array}$} & \multicolumn{2}{|c|}{$\begin{array}{c}\text { Dose Journalière d'Exposition } \\
\text { DJE }(\mathrm{mg} / \mathrm{kg} / \mathrm{j})\end{array}$} & \multirow{2}{*}{$\begin{array}{l}\text { Dose Journalière } \\
\text { Admise DJA } \\
\text { (mg/kg/j) du Cd }\end{array}$} & \multicolumn{2}{|c|}{$\begin{array}{c}\text { Risque sanitaire } \\
\text { (Quotient de danger) }\end{array}$} \\
\hline & & & & Adulte & Enfant & Adulte & Enfant & & Adulte & Enfant \\
\hline Kessounou & 0,003 & 0,03766 & 0,090384 & \multirow{8}{*}{70} & \multirow{8}{*}{28} & 3,87E-06 & $9,68 \mathrm{E}-06$ & \multirow{8}{*}{$2,00 \mathrm{E}-04$} & 0,02 & 0,05 \\
\hline Hozin & 0,044 & 0,03569 & 0,085656 & & & $5,38 \mathrm{E}-05$ & $1,35 \mathrm{E}-04$ & & 0,27 & 0,67 \\
\hline Akpadanou & 0,008 & 0,03974 & 0,095376 & & & $1,09 \mathrm{E}-05$ & 2,73E-05 & & 0,05 & 0,14 \\
\hline Todé & 0,015 & 0,06923 & 0,166152 & & & $3,56 \mathrm{E}-05$ & $8,90 \mathrm{E}-05$ & & 0,18 & 0,45 \\
\hline Affamè & 0,034 & 0,12051 & 0,289224 & & & $1,40 \mathrm{E}-04$ & 3,51E-04 & & 0,70 & 1,76 \\
\hline Agonhoui & 0,067 & 0,19268 & 0,462432 & & & $4,43 \mathrm{E}-04$ & $1,11 \mathrm{E}-03$ & & 2,21 & 5,53 \\
\hline Bembè & 0,017 & 0,04073 & 0,097752 & & & $2,37 \mathrm{E}-05$ & $5,93 \mathrm{E}-05$ & & 0,12 & 0,30 \\
\hline Houinta & 0,017 & 0,03785 & 0,09084 & & & $2,21 \mathrm{E}-05$ & $5,52 \mathrm{E}-05$ & & 0,11 & 0,28 \\
\hline
\end{tabular}

Tableau 2: Risques sanitaires liés à la consommation d'escargots Archachatina marginata frits contaminés par le Pb dans la vallée de l'Ouémé.

\begin{tabular}{|c|c|c|c|c|c|c|c|c|c|c|}
\hline \multirow{2}{*}{ Plomb } & \multirow{2}{*}{$\begin{array}{c}\text { Concentration } \\
\text { moyenne en métaux } \\
\text { lourds C (mg/Kg) }\end{array}$} & \multirow{2}{*}{$\begin{array}{l}\text { Poids moyen } \\
\text { des escargots } \\
(\mathrm{kg})\end{array}$} & \multirow{2}{*}{$\begin{array}{c}\text { Quantité } \\
\text { d'escargots } \\
\text { ingérés par jour Q } \\
(\mathrm{Kg} / \mathbf{j})\end{array}$} & \multicolumn{2}{|c|}{$\begin{array}{c}\text { Poids de la cible } \mathbf{P} \\
(\mathrm{kg})\end{array}$} & \multicolumn{2}{|c|}{$\begin{array}{c}\text { Dose Journalière } \\
\text { d'Exposition DJE (mg/kg/j) }\end{array}$} & \multirow{2}{*}{$\begin{array}{c}\text { Dose Journalière } \\
\text { Admise DJA (mg/kg/j) } \\
\text { du Pb }\end{array}$} & \multicolumn{2}{|c|}{$\begin{array}{c}\text { Risque sanitaire } \\
\text { (Quotient de danger) }\end{array}$} \\
\hline & & & & Adulte & Enfant & Adulte & Enfant & & Adulte & Enfant \\
\hline Kessounou & 0,062 & 0,03766 & 0,090384 & \multirow{8}{*}{70} & \multirow{8}{*}{28} & $8,01 \mathrm{E}-05$ & $2,00 \mathrm{E}-04$ & \multirow{8}{*}{$3,60 \mathrm{E}-03$} & 0,02 & 0,06 \\
\hline Hozin & 0,028 & 0,03569 & 0,085656 & & & $3,43 \mathrm{E}-05$ & $8,57 \mathrm{E}-05$ & & 0,01 & 0,02 \\
\hline Akpadanou & 0,045 & 0,03974 & 0,095376 & & & $6,13 \mathrm{E}-05$ & $1,53 \mathrm{E}-04$ & & 0,02 & 0,04 \\
\hline Todé & 0,029 & 0,06923 & 0,166152 & & & $6,88 \mathrm{E}-05$ & $1,72 \mathrm{E}-04$ & & 0,02 & 0,05 \\
\hline Affamè & 0,061 & 0,12051 & 0,289224 & & & $2,52 \mathrm{E}-04$ & $6,30 \mathrm{E}-04$ & & 0,07 & 0,18 \\
\hline Agonhoui & 0,056 & 0,19268 & 0,462432 & & & $3,70 \mathrm{E}-04$ & $9,25 \mathrm{E}-04$ & & 0,10 & 0,26 \\
\hline Bembè & 0,023 & 0,04073 & 0,097752 & & & $3,21 \mathrm{E}-05$ & $8,03 \mathrm{E}-05$ & & 0,01 & 0,02 \\
\hline Houinta & 0,025 & 0,03785 & 0,09084 & & & $3,24 \mathrm{E}-05$ & $8,11 \mathrm{E}-05$ & & 0,01 & 0,02 \\
\hline
\end{tabular}


M. R. ADAMOU et al. / Int. J. Biol. Chem. Sci. 13(1): 126-143, 2019

Tableau 3: Risques sanitaires liés à la consommation d'escargots Archachatina marginata bouillis contaminés par le Cd dans la vallée de l'Ouémé.

\begin{tabular}{|c|c|c|c|c|c|c|c|c|c|c|}
\hline \multirow{2}{*}{ Cadmium } & \multirow{2}{*}{$\begin{array}{c}\text { Concentration } \\
\text { moyenne en } \\
\text { métaux lourds C } \\
(\mathrm{mg} / \mathrm{Kg})\end{array}$} & \multirow{2}{*}{$\begin{array}{c}\text { Poids moyen } \\
\text { des escargots } \\
(\mathrm{kg}) \\
\end{array}$} & \multirow{2}{*}{$\begin{array}{c}\text { Quantité } \\
\text { d'escargots } \\
\text { ingérés par jour Q } \\
(\mathrm{Kg} / \mathbf{j})\end{array}$} & \multicolumn{2}{|c|}{$\begin{array}{c}\text { Poids de la cible } P \\
(\mathbf{k g})\end{array}$} & \multicolumn{2}{|c|}{$\begin{array}{c}\text { Dose Journalière } \\
\text { d'Exposition DJE (mg/kg/j) }\end{array}$} & \multirow{2}{*}{$\begin{array}{c}\text { Dose Journalière } \\
\text { Admise DJA } \\
(\mathrm{mg} / \mathrm{kg} / \mathbf{j}) \text { du Cd }\end{array}$} & \multicolumn{2}{|c|}{$\begin{array}{c}\text { Risque sanitaire } \\
\text { (Quotient de danger) }\end{array}$} \\
\hline & & & & Adulte & Enfant & Adulte & Enfant & & Adulte & Enfant \\
\hline Kessounou & 0,002 & 0,03766 & 0,090384 & \multirow{8}{*}{70} & \multirow{8}{*}{28} & $2,58 \mathrm{E}-06$ & $6,46 \mathrm{E}-06$ & \multirow{8}{*}{$2,00 \mathrm{E}-04$} & 0,01 & 0,03 \\
\hline Hozin & 0,035 & 0,03569 & 0,085656 & & & $4,28 \mathrm{E}-05$ & 1,07E-04 & & 0,21 & 0,54 \\
\hline Akpadanou & 0,006 & 0,03974 & 0,095376 & & & $8,18 \mathrm{E}-06$ & $2,04 \mathrm{E}-05$ & & 0,04 & 0,10 \\
\hline Todé & 0,013 & 0,06923 & 0,166152 & & & $3,09 \mathrm{E}-05$ & $7,71 \mathrm{E}-05$ & & 0,15 & 0,39 \\
\hline Affamè & 0,043 & 0,12051 & 0,289224 & & & $1,78 \mathrm{E}-04$ & 4,44E-04 & & 0,89 & 2,22 \\
\hline Agonhoui & 0,046 & 0,19268 & 0,462432 & & & $3,04 \mathrm{E}-04$ & $7,60 \mathrm{E}-04$ & & 1,52 & 3,80 \\
\hline Bembè & 0,001 & 0,04073 & 0,097752 & & & $1,40 \mathrm{E}-06$ & $3,49 \mathrm{E}-06$ & & 0,01 & 0,02 \\
\hline Houinta & 0,022 & 0,03785 & 0,09084 & & & $2,85 \mathrm{E}-05$ & $7,14 \mathrm{E}-05$ & & 0,14 & 0,36 \\
\hline
\end{tabular}

Tableau 4 : Risques sanitaires liés à la consommation d'escargots bouillis Archachatina marginata contaminés par le Pb dans la vallée de l'Ouémé.

\begin{tabular}{|c|c|c|c|c|c|c|c|c|c|c|}
\hline \multirow{2}{*}{ Plomb } & \multirow{2}{*}{$\begin{array}{c}\text { Concentration } \\
\text { moyenne en métaux } \\
\text { lourds C (mg/Kg) }\end{array}$} & \multirow{2}{*}{$\begin{array}{l}\text { Poids moyen } \\
\text { des escargots } \\
\quad(\mathrm{kg})\end{array}$} & \multirow{2}{*}{$\begin{array}{c}\text { Quantité } \\
\text { d'escargots } \\
\text { ingérés par } \\
\text { jour } Q(\mathrm{Kg} / \mathbf{j})\end{array}$} & \multicolumn{2}{|c|}{$\begin{array}{c}\text { Poids de la cible } \mathbf{P} \\
(\mathrm{kg})\end{array}$} & \multicolumn{2}{|c|}{$\begin{array}{c}\text { Dose Journalière } \\
\text { d'Exposition DJE (mg/kg/j) } \\
\end{array}$} & \multirow[b]{2}{*}{$\begin{array}{c}\text { Dose Journalière Admise } \\
\text { DJA }(\mathrm{mg} / \mathrm{kg} / \mathrm{j}) \text { du Pb }\end{array}$} & \multicolumn{2}{|c|}{$\begin{array}{c}\text { Risque sanitaire } \\
\text { (Quotient de danger) }\end{array}$} \\
\hline & & & & Adulte & Enfant & Adulte & Enfant & & Adulte & Enfant \\
\hline Kessounou & 0,049 & 0,03766 & 0,090384 & \multirow{8}{*}{70} & \multirow{8}{*}{28} & $6,33 \mathrm{E}-05$ & $1,58 \mathrm{E}-04$ & \multirow{8}{*}{$3,60 \mathrm{E}-03$} & 0,02 & 0,04 \\
\hline Hozin & 0,046 & 0,03569 & 0,085656 & & & $5,63 \mathrm{E}-05$ & $1,41 \mathrm{E}-04$ & & 0,02 & 0,04 \\
\hline Akpadanou & 0,075 & 0,03974 & 0,095376 & & & $1,02 \mathrm{E}-04$ & $2,55 \mathrm{E}-04$ & & 0,03 & 0,07 \\
\hline Todé & 0,054 & 0,06923 & 0,166152 & & & $1,28 \mathrm{E}-04$ & $3,20 \mathrm{E}-04$ & & 0,04 & 0,09 \\
\hline Affamè & 0,076 & 0,12051 & 0,289224 & & & $3,14 \mathrm{E}-04$ & $7,85 \mathrm{E}-04$ & & 0,09 & 0,22 \\
\hline Agonhoui & 0,062 & 0,19268 & 0,462432 & & & $4,10 \mathrm{E}-04$ & $1,02 \mathrm{E}-03$ & & 0,11 & 0,28 \\
\hline Bembè & 0,096 & 0,04073 & 0,097752 & & & $1,34 \mathrm{E}-04$ & $3,35 \mathrm{E}-04$ & & 0,04 & 0,09 \\
\hline Houinta & 0,035 & 0,03785 & 0,09084 & & & $4,54 \mathrm{E}-05$ & $1,14 \mathrm{E}-04$ & & 0,01 & 0,03 \\
\hline
\end{tabular}


Tableau 5 : Risques sanitaires liés à la consommation d'escargots Limicolaria spp frits contaminés par le Cd dans la vallée de l'Ouémé.

\begin{tabular}{|c|c|c|c|c|c|c|c|c|c|c|}
\hline \multirow{2}{*}{ Cadmium } & \multirow{2}{*}{$\begin{array}{c}\text { Concentration } \\
\text { moyenne en métaux } \\
\text { lourds } \mathrm{C}(\mathrm{mg} / \mathrm{Kg})\end{array}$} & \multirow{2}{*}{$\begin{array}{c}\text { Poids moyen } \\
\text { des escargots } \\
(\mathrm{kg})\end{array}$} & \multirow{2}{*}{$\begin{array}{c}\text { Quantité } \\
\text { d'escargots } \\
\text { ingérés par jour Q } \\
(\mathrm{Kg} / \mathbf{j})\end{array}$} & \multicolumn{2}{|c|}{$\begin{array}{c}\text { Poids de la cible } P \\
(\mathbf{k g})\end{array}$} & \multicolumn{2}{|c|}{$\begin{array}{c}\text { Dose Journalière } \\
\text { d'Exposition DJE (mg/kg/j) }\end{array}$} & \multirow{2}{*}{$\begin{array}{c}\text { Dose Journalière } \\
\text { Admise DJA } \\
(\mathrm{mg} / \mathrm{kg} / \mathrm{j}) \text { du Cd }\end{array}$} & \multicolumn{2}{|c|}{$\begin{array}{c}\text { Risque sanitaire } \\
\text { (Quotient de danger) }\end{array}$} \\
\hline & & & & Adulte & Enfant & Adulte & Enfant & & Adulte & Enfant \\
\hline Kessounou & 0,769 & 0,00376 & 0,018048 & \multirow{8}{*}{70} & \multirow{8}{*}{28} & $1,98 \mathrm{E}-04$ & $4,96 \mathrm{E}-04$ & \multirow{8}{*}{$2,00 \mathrm{E}-04$} & 0,99 & 2,48 \\
\hline Hozin & 0 & 0,00623 & 0,029904 & & & $0,00 \mathrm{E}+00$ & $0,00 \mathrm{E}+00$ & & 0,00 & 0,00 \\
\hline Akpadanou & 0,015 & 0,01002 & 0,048096 & & & $1,03 \mathrm{E}-05$ & $2,58 \mathrm{E}-05$ & & 0,05 & 0,13 \\
\hline Todé & 0,458 & 0,00339 & 0,016272 & & & $1,06 \mathrm{E}-04$ & $2,66 \mathrm{E}-04$ & & 0,53 & 1,33 \\
\hline Affamè & 0,088 & 0,00852 & 0,040896 & & & $5,14 \mathrm{E}-05$ & $1,29 \mathrm{E}-04$ & & 0,26 & 0,64 \\
\hline Agonhoui & 0,062 & 0,00464 & 0,022272 & & & $1,97 \mathrm{E}-05$ & $4,93 \mathrm{E}-05$ & & 0,10 & 0,25 \\
\hline Bembè & 0,16 & 0,00616 & 0,029568 & & & $6,76 \mathrm{E}-05$ & $1,69 \mathrm{E}-04$ & & 0,34 & 0,84 \\
\hline Houinta & 0 & 0,00588 & 0,028224 & & & $0,00 \mathrm{E}+00$ & $0,00 \mathrm{E}+00$ & & 0,00 & 0,00 \\
\hline
\end{tabular}

Tableau 6: Risques sanitaires liés à la consommation d'escargots Limicolaria spp frits contaminés par le Pb dans la vallée de l'Ouémé.

\begin{tabular}{|c|c|c|c|c|c|c|c|c|c|c|}
\hline \multirow[t]{2}{*}{ Plomb } & \multirow{2}{*}{$\begin{array}{c}\text { Concentration } \\
\text { moyenne en métaux } \\
\text { lourds C (mg/Kg) }\end{array}$} & \multirow{2}{*}{$\begin{array}{l}\text { Poids moyen } \\
\text { des escargots } \\
\text { (kg) }\end{array}$} & \multirow{2}{*}{$\begin{array}{c}\text { Quantité } \\
\text { d'escargots } \\
\text { ingérés par jour Q } \\
(\mathrm{Kg} / \mathbf{j})\end{array}$} & \multicolumn{2}{|c|}{$\begin{array}{c}\text { Poids de la cible } P \\
(\mathrm{~kg})\end{array}$} & \multicolumn{2}{|c|}{$\begin{array}{c}\text { Dose Journalière } \\
\text { d'Exposition DJE } \\
(\mathrm{mg} / \mathrm{kg} / \mathrm{j})\end{array}$} & \multirow{2}{*}{$\begin{array}{c}\text { Dose Journalière } \\
\text { Admise DJA } \\
\text { (mg/kg/j) du Pb }\end{array}$} & \multicolumn{2}{|c|}{$\begin{array}{c}\text { Risque sanitaire } \\
\text { (Quotient de danger) }\end{array}$} \\
\hline & & & & Adulte & Enfant & Adulte & Enfant & & Adulte & Enfant \\
\hline Kessounou & 0,275 & 0,00376 & 0,018048 & \multirow{8}{*}{70} & \multirow{8}{*}{28} & $7,09 \mathrm{E}-05$ & $1,77 \mathrm{E}-04$ & \multirow{8}{*}{$3,60 \mathrm{E}-03$} & 0,02 & 0,05 \\
\hline Hozin & 0 & 0,00623 & 0,029904 & & & $0,00 \mathrm{E}+00$ & $0,00 \mathrm{E}+00$ & & 0,00 & 0,00 \\
\hline Akpadanou & 0,206 & 0,01002 & 0,048096 & & & $1,42 \mathrm{E}-04$ & $3,54 \mathrm{E}-04$ & & 0,04 & 0,10 \\
\hline Todé & 0,094 & 0,00339 & 0,016272 & & & $2,19 \mathrm{E}-05$ & $5,46 \mathrm{E}-05$ & & 0,01 & 0,02 \\
\hline Affamè & 0,118 & 0,00852 & 0,040896 & & & $6,89 \mathrm{E}-05$ & $1,72 \mathrm{E}-04$ & & 0,02 & 0,05 \\
\hline Agonhoui & 0,147 & 0,00464 & 0,022272 & & & $4,68 \mathrm{E}-05$ & $1,17 \mathrm{E}-04$ & & 0,01 & 0,03 \\
\hline Bembè & 0,123 & 0,00616 & 0,029568 & & & $5,20 \mathrm{E}-05$ & $1,30 \mathrm{E}-04$ & & 0,01 & 0,04 \\
\hline Houinta & 0,075 & 0,00588 & 0,028224 & & & $3,02 \mathrm{E}-05$ & $7,56 \mathrm{E}-05$ & & 0,01 & 0,02 \\
\hline
\end{tabular}


Tableau 7 : Risques sanitaires liés à la consommation d'escargots Limicolaria spp bouillis contaminés par le Cd dans la vallée de l'Ouémé.

\begin{tabular}{|c|c|c|c|c|c|c|c|c|c|c|}
\hline \multirow{2}{*}{ Cadmium } & \multirow{2}{*}{$\begin{array}{c}\text { Concentration } \\
\text { moyenne en métaux } \\
\text { lourds } \mathrm{C}(\mathrm{mg} / \mathrm{Kg})\end{array}$} & \multirow{2}{*}{$\begin{array}{l}\text { Poids moyen } \\
\text { des escargots } \\
\text { (kg) }\end{array}$} & \multirow{2}{*}{$\begin{array}{c}\text { Quantité } \\
\text { d'escargots } \\
\text { ingérés par jour Q } \\
(\mathrm{Kg} / \mathbf{j})\end{array}$} & \multicolumn{2}{|c|}{$\begin{array}{c}\text { Poids de la cible } P \\
(\mathrm{~kg})\end{array}$} & \multicolumn{2}{|c|}{$\begin{array}{c}\text { Dose Journalière d'Exposition } \\
\text { DJE }(\mathrm{mg} / \mathrm{kg} / \mathrm{j})\end{array}$} & \multirow{2}{*}{$\begin{array}{c}\text { Dose Journalière } \\
\text { Admise DJA } \\
(\mathrm{mg} / \mathrm{kg} / \mathbf{j}) \text { du Cd }\end{array}$} & \multicolumn{2}{|c|}{$\begin{array}{c}\text { Risque sanitaire } \\
\text { (Quotient de danger) }\end{array}$} \\
\hline & & & & Adulte & Enfant & Adulte & Enfant & & Adulte & Enfant \\
\hline Kessounou & 0,151 & 0,00376 & 0,018048 & \multirow{8}{*}{70} & \multirow{8}{*}{28} & $3,89 \mathrm{E}-05$ & $9,73 \mathrm{E}-05$ & \multirow{8}{*}{$2,00 \mathrm{E}-04$} & 0,19 & 0,49 \\
\hline Hozin & 0 & 0,00623 & 0,029904 & & & $0,00 \mathrm{E}+00$ & $0,00 \mathrm{E}+00$ & & 0,00 & 0,00 \\
\hline Akpadanou & 0 & 0,01002 & 0,048096 & & & $0,00 \mathrm{E}+00$ & $0,00 \mathrm{E}+00$ & & 0,00 & 0,00 \\
\hline Todé & 0,422 & 0,00339 & 0,016272 & & & $9,81 \mathrm{E}-05$ & $2,45 \mathrm{E}-04$ & & 0,49 & 1,23 \\
\hline Affamè & 0,06 & 0,00852 & 0,040896 & & & $3,51 \mathrm{E}-05$ & $8,76 \mathrm{E}-05$ & & 0,18 & 0,44 \\
\hline Agonhoui & 0,059 & 0,00464 & 0,022272 & & & $1,88 \mathrm{E}-05$ & $4,69 \mathrm{E}-05$ & & 0,09 & 0,23 \\
\hline Bembè & 0,071 & 0,00616 & 0,029568 & & & $3,00 \mathrm{E}-05$ & $7,50 \mathrm{E}-05$ & & 0,15 & 0,37 \\
\hline Houinta & 0,412 & 0,00588 & 0,028224 & & & $1,66 \mathrm{E}-04$ & $4,15 \mathrm{E}-04$ & & 0,83 & 2,08 \\
\hline
\end{tabular}

Tableau 8: Risques sanitaires liés à la consommation d'escargots bouillis Limicolaria spp contaminés par le Pb dans la vallée de l'Ouémé.

\begin{tabular}{|c|c|c|c|c|c|c|c|c|c|c|}
\hline \multirow[t]{2}{*}{ Plomb } & \multirow{2}{*}{$\begin{array}{l}\text { Concentration } \\
\text { moyenne en métaux } \\
\text { lourds } \mathrm{C}(\mathrm{mg} / \mathrm{Kg})\end{array}$} & \multirow{2}{*}{$\begin{array}{l}\text { Poids moyen } \\
\text { des escargots } \\
\text { (kg) }\end{array}$} & \multirow{2}{*}{$\begin{array}{c}\text { Quantité } \\
\text { d'escargots } \\
\text { ingérés par jour Q } \\
(\mathrm{Kg} / \mathbf{j})\end{array}$} & \multicolumn{2}{|c|}{$\begin{array}{c}\text { Poids de la cible } P \\
(\mathrm{~kg})\end{array}$} & \multicolumn{2}{|c|}{$\begin{array}{c}\text { Dose Journalière } \\
\text { d'Exposition DJE } \\
(\mathrm{mg} / \mathrm{kg} / \mathrm{j})\end{array}$} & \multirow{2}{*}{$\begin{array}{l}\text { Dose Journalière } \\
\text { Admise DJA } \\
(\mathrm{mg} / \mathrm{kg} / \mathrm{j}) \mathrm{du} \mathrm{Pb}\end{array}$} & \multicolumn{2}{|c|}{$\begin{array}{c}\text { Risque sanitaire } \\
\text { (Quotient de danger) }\end{array}$} \\
\hline & & & & Adulte & Enfant & Adulte & Enfant & & Adulte & Enfant \\
\hline Kessounou & 1,024 & 0,00376 & 0,018048 & \multirow{8}{*}{70} & \multirow{8}{*}{28} & $2,64 \mathrm{E}-04$ & $6,60 \mathrm{E}-04$ & \multirow{8}{*}{$3,60 \mathrm{E}-03$} & 0,07 & 0,18 \\
\hline Hozin & 0,088 & 0,00623 & 0,029904 & & & $3,76 \mathrm{E}-05$ & $9,40 \mathrm{E}-05$ & & 0,01 & 0,03 \\
\hline Akpadanou & 0,153 & 0,01002 & 0,048096 & & & $1,05 \mathrm{E}-04$ & $2,63 \mathrm{E}-04$ & & 0,03 & 0,07 \\
\hline Todé & 0,05 & 0,00339 & 0,016272 & & & $1,16 \mathrm{E}-05$ & $2,91 \mathrm{E}-05$ & & 0,00 & 0,01 \\
\hline Affamè & 0,146 & 0,00852 & 0,040896 & & & $8,53 \mathrm{E}-05$ & $2,13 \mathrm{E}-04$ & & 0,02 & 0,06 \\
\hline Agonhoui & 0,188 & 0,00464 & 0,022272 & & & $5,98 \mathrm{E}-05$ & $1,50 \mathrm{E}-04$ & & 0,02 & 0,04 \\
\hline Bembè & 0,016 & 0,00616 & 0,029568 & & & $6,76 \mathrm{E}-06$ & $1,69 \mathrm{E}-05$ & & 0,00 & 0,00 \\
\hline Houinta & 0,354 & 0,00588 & 0,028224 & & & $1,43 \mathrm{E}-04$ & $3,57 \mathrm{E}-04$ & & 0,04 & 0,10 \\
\hline
\end{tabular}


M. R. ADAMOU et al. / Int. J. Biol. Chem. Sci. 13(1): 126-143, 2019

Tableau 9: Résultats du test t de Student sur le logarithme népérien des valeurs individuelles de risque sanitaire chez les adultes et les enfants.

\begin{tabular}{|c|c|c|c|c|c|}
\hline \multirow{2}{*}{ Formes } & \multicolumn{2}{|c|}{ Adultes } & \multicolumn{2}{|c|}{ Enfants } & \multirow{2}{*}{ Probabilité } \\
\hline & Moyenne & $\operatorname{cv}(\%)$ & Moyenne & $\operatorname{cv}(\%)$ & \\
\hline Cadmium & 0.41 & 152.34 & 1.04 & 152.34 & 0.041 \\
\hline Plomb & 0.04 & 92.84 & 0.09 & 92.84 & 0.163 \\
\hline Probabilité & 0.004 & - & 0.004 & - & \\
\hline Bouilli & 0.21 & 197.65 & 0.52 & 197.65 & 0.381 \\
\hline Frit & 0.24 & 226.17 & 0.61 & 226.17 & 0.306 \\
\hline Probabilité & 0.999 & - & 0.999 & - & - \\
\hline
\end{tabular}




\section{DISCUSSION}

\section{Concentrations des métaux lourds dans les escargots}

Le cadmium et le plomb après les analyses ont été observés dans les deux espèces d'escargots bouillis et frits. Dans les escargots bouillis, les concentrations moyennes de plomb obtenues après avoir bouilli Archachatina marginata sont très supérieures dans toutes les localités aux valeurs toxicologiques de référence fixées par l'OMS (normes journalières) pour l'exposition à ce métal par voie orale. Ces concentrations dépassent jusqu'à 27 fois la norme à Bembè. Cette grande supériorité des concentrations moyennes de plomb est aussi observée pour Limicolaria spp bouilli dans toutes les localités où les teneurs enregistrées vont jusqu'à 292 fois la norme à Kessounou. Les concentrations moyennes de cadmium obtenues après avoir bouilli Archachatina marginata sont très supérieures aux valeurs toxicologiques de référence fixées par l'ATDSR (normes journalières) pour l'exposition à ce métal par voie orale dans toutes les localités à l'exception de Kessounou et de Bembè. Les concentrations moyennes enregistrées dépassent jusqu'à 230 fois la norme à Agonhoui. Pour Limicolaria spp bouilli, cette grande supériorité des concentrations moyennes de cadmium est aussi observée dans toutes les localités à l'exception de Hozin et d'Akpadanou. A Todé, la concentration moyenne enregistrée dépasse 2110 fois la norme et à Houinta 2060 fois. On peut déduire de ces résultats qu'il pourrait y avoir un risque sanitaire lié à la consommation des escargots bouillis de la vallée de l'Ouémé. Ces résultats sont différents de ceux obtenus par Kobia et al. (2016) sur la viande d'animaux de brousse, en l'occurrence le rat géant. Les concentrations moyennes de plomb $(13,93 \mathrm{mg} / \mathrm{kg})$ qu'ils ont enregistré dans la viande bouillie dépassent largement celles obtenues dans cette étude dans toutes les localités pour toutes les deux espèces. Par contre les concentrations moyennes de cadmium enregistrées $(0,10$ $\mathrm{mg} / \mathrm{kg}$ ) sont inférieures à celles enregistrées dans cette étude à Kessounou, Todé et Houinta pour Limicolaria spp.

Dans les escargots frits, les concentrations moyennes de plomb obtenues après avoir frit Archachatina marginata sont très supérieures dans toutes les localités aux valeurs toxicologiques de référence fixées par l'OMS (normes journalières) pour l'exposition à ce métal par voie orale. Ces concentrations dépassent jusqu'à 17 fois la norme à Kessounou et à Agonhoui. A l'exception de Hozin, cette grande supériorité des concentrations moyennes de plomb est aussi observée pour Limicolaria spp frit dans toutes les localités où les teneurs enregistrées vont jusqu'à 78 fois la norme à Kessounou. Les concentrations moyennes de cadmium obtenues après avoir frit Archachatina marginata sont très supérieures aux valeurs toxicologiques de référence fixées par l'ATDSR (normes journalières) pour l'exposition à ce métal par voie orale dans toutes les localités à l'exception de Kessounou. Les concentrations moyennes enregistrées dépassent jusqu'à 335 fois la norme à Agonhoui. Pour Limicolaria spp frit, cette grande supériorité de concentrations moyennes de cadmium est aussi observée dans toutes les localités à l'exception de Hozin et de Houinta. A Kessounou, la concentration moyenne enregistrée dépasse 3485 fois la norme et à Todé 2290 fois. On peut déduire de ces résultats qu'il pourrait y avoir un risque sanitaire lié à la consommation des escargots frits de la vallée de l'Ouémé. Ces résultats sont largement inférieurs dans presque toutes les localités pour Archachatina marginata à ceux obtenus par Tawfik (2013) dans le mullet (Mugil cephalus) et la sardine (Sardinops sagax) frits où il a enregistré pour le cadmium respectivement $0,360 \mu \mathrm{g} / \mathrm{g}$ et $0,821 \mu \mathrm{g} / \mathrm{g}$, et pour le plomb respectivement $0,011 \mu \mathrm{g} / \mathrm{g}$ et $0,022 \mu \mathrm{g} / \mathrm{g}$. Par contre les concentrations enregistrées dans cette étude pour Limicolaria spp dépassent dans plusieurs localités celles obtenues par Tawfik (2013) pour le cadmium et le plomb. Les résultats de 
la présente étude diffèrent aussi de ceux obtenus par Czech et Stachyra (2012) dans la crevette Penaeus setiferus $\left(0,100 \mathrm{mg} \mathrm{Cd} \cdot \mathrm{kg}^{-1}\right.$ et $\left.0,057 \mathrm{mg} \mathrm{Pb} \cdot \mathrm{kg}^{-1}\right)$, le calmar Todarodes pacificus $\left(0,704 \mathrm{mg}\right.$ Cd. $\mathrm{kg}^{-1}$ et $0,140 \mathrm{mg}$ Pb.kg $\left.{ }^{-1}\right)$, la moule Mytilus edulis $\quad(0,190 \mathrm{mg}$ Cd. $\mathrm{kg}^{-1}$ et $\left.0,059 \mathrm{mg} \mathrm{Pb} \cdot \mathrm{kg}^{-1}\right)$ et la pieuvre Octopus vulgaris $\left(1,913 \mathrm{mg} \mathrm{Cd} \cdot \mathrm{kg}^{-1}\right.$ et 0,401 $\left.\mathrm{mg} \mathrm{Pb} . \mathrm{kg}^{-1}\right)$. Ces teneurs sont largement supérieures à celles enregistrées dans cette étude pour Archachatina marginata dans presque toutes les localités. Elles sont par contre inférieures dans certaines localités aux teneurs enregistrées pour Limicolaria spp.

\section{Risques sanitaires liés à la consommation des escargots contaminés aux métaux lourds}

L'évaluation du risque sanitaire lié à la consommation des escargots contaminés aux métaux lourds montre que les quotients de danger issus des escargots bouillis et frits pour les enfants sont tous supérieurs à ceux des adultes dans toutes les localités. Pour le cadmium, les quotients de danger sont supérieurs à 1 chez les adultes et les enfants dans plusieurs localités pour l'espèce Archachatina marginata. Quant à l'espèce Limicolaria spp, ils sont supérieurs à 1 dans plusieurs localités uniquement chez les enfants. Ces résultats s'apparentent de ceux obtenus par Ouorou-Sama et al. (2014). Ils ont en effet enregistré que la survenue d'un effet toxique provenant du cadmium et lié à la consommation des poissons du lac Togo est peu probable $(\mathrm{QD}=0,81<1)$ chez les adultes. Cependant, l'on peut s'attendre à des effets toxiques chez les enfants $(\mathrm{QD}=2,04>1)$. Aussi ont-ils noté qu'il n'y a pas de risque d'effets toxiques lié au plomb en ce qui concerne la consommation des poissons du lac aussi bien chez les adultes ( $\mathrm{QD}=0,16<1$ ) que chez les enfants $(\mathrm{QD}=0,40<1)$. Les enfants sont donc les plus exposés aux métaux lourds. Ce que confirme Hounkpatin et al. (2012) qui le justifie à leur faible poids corporel et à leur fragilité sur le plan physiologique étant donné que les contaminants sont facilement absorbés dans leur organisme. En effet, l'organisme des enfants absorbe potentiellement plus de contaminants et reste incapable de les éliminer aussi facilement que les adultes étant donné que leurs systèmes d'élimination sont moins développés (Hounkpatin et al., 2012). Ces résultats révèlent donc que la consommation de 8 escargots Archachatina marginata et de 16 escargots Limicolaria spp provenant de la vallée de l'Ouémé au Bénin présenterait un danger pour la santé des adultes et encore plus des enfants à cause de l'accumulation $\mathrm{du} \mathrm{Cd}$ dans la chair des escargots malgré la cuisson. Pourtant, le cadmium n'a aucun rôle métabolique connu et ne semble pas biologiquement essentiel ou bénéfique au métabolisme des êtres vivants (Miquel, 2001). Il est un toxique cumulatif dont la demi-vie biologique est de l'ordre de 20 à 30 ans. L'exposition chronique au cadmium entraine l'apparition d'une néphropathie irréversible pouvant évoluer vers une insuffisance rénale (Bisson et al., 2011). Chez l'homme, sa toxicité aigüe est connue depuis 1950 sous le nom du syndrome d'Itaï-Itaï défini par l'association d'une insuffisance rénale avec ostéoporose (déminéralisation et fragilisation des os) et ostéomalacie (déminéralisation et déformation des os) avec des douleurs osseuses intenses (Bliefert et Perraud, 2008). Le cadmium est cancérigène et tératogène (Satoh et al., 2002 ; Banerjee et Flores-Rozas, 2005).

Pour réduire le risque sanitaire dû au cadmium, il faut pour les escargots Archachatina marginata bouillis une consommation de 5 individus au plus par repas chez les adultes et de 2 chez les enfants. Et pour les frits, il faut 3 individus par repas chez les adultes et 1 chez les enfants. Par contre pour les escargots Limicolaria spp bouillis, il faut chez les enfants une consommation de 7 individus au plus par repas et pour les frits une consommation de 6 au plus. Ces résultats diffèrent de ceux obtenus par Edorh et al. (2009) qui ont observé que la consommation de seulement 
$100 \mathrm{~g}$ de viande de l'escargot Archachatina marginata par jour empoisonnera le consommateur avec $856 \mu \mathrm{g}$ de plomb et 124 $\mu \mathrm{g}$ de cadmium, qui dépassent déjà largement la dose journalière admise qui est respectivement de $234 \mu \mathrm{g}$ et $124 \mu \mathrm{g}$ pour un adulte de $65 \mathrm{~kg}$. Seule une personne qui a mangé $27 \mathrm{mg}$ d'escargot par jour est protégée contre toute exposition, au moins en ce qui concerne le plomb d'après leur étude. Toutefois, il est important de préciser que le dépassement de la DJA n'implique pas nécessairement une pathologie, car les nourritures contiennent aussi des substances capables de s'opposer à la distribution des métaux toxiques dans le corps et à ses effets. Ce sont des antioxydants, des polyphénols, du soufre et des métaux chélateurs naturels (Edorh et al., 2009).

\section{Conclusion}

$\mathrm{Au}$ total, les escargots Archachatina marginata et Limicolaria spp bouillis et frits de la vallée de l'Ouémé ont concentré des métaux lourds comme le cadmium et le plomb au-delà des normes admises. L'évaluation des risques sanitaires a révélé que la consommation des escargots provenant surtout de la commune de Bonou présenterait un danger pour la santé des adultes et des enfants à cause de l'accumulation du cadmium dans la viande des escargots malgré la cuisson. Il urge donc que les populations réduisent la quantité d'escargots consommés par repas ainsi que la fréquence. Elles doivent aussi privilégier la consommation des aliments renfermant des antioxydants afin de se garantir une meilleure santé. L'étude n'a pas pu prendre en compte les enquêtes sur les signes cliniques liés au cadmium ni évaluer la cadmiumémie et la plombémie chez les populations. Ces aspects feront l'objet des prochains travaux scientifiques.

\section{CONFLIT D'INTERETS}

Les auteurs déclarent qu'il n'y a aucun conflit d'intérêts.

\section{CONTRIBUTIONS DES AUTEURS}

Ce travail est le fruit de la contribution de tous les auteurs. MRA a conçu le questionnaire d'enquête, réalisé les manipulations au laboratoire, analysé les données obtenues et a rédigé le manuscrit avec AYD. EYP a validé la fiche d'enquête et les protocoles de laboratoire, contribué à l'amélioration du manuscrit et supervisé tous les travaux.

\section{REFERENCES}

ASTEE (Association Scientifique et Technique de l'Eau et de l'Environnement). 2003. Guide pour l'évaluation du risque sanitaire dans le cadre de l'étude d'impact d'une UIOM. ASTEE, $60 \mathrm{p}$.

Banerjee S, Flores-Rozas H. 2005. Cadmium inhibits mismatch repair by blocking the ATPase activity of the MSH2-MSH6 complex. Nucleic. Acids Res., 33: 14101419. DOI: $10.1093 /$ nar/gki291

Bassey FI, Oguntunde FC, Iwegbue CMA, Osabor VN, Edem CA. 2014. Effects of processing on the proximate and metal contents in three fish species from Nigerian coastal waters. Food Sci Nutr., 2(3) : 272-281. DOI: 10.1002/fsn3.102

Bisson M, Diderich R, Houeix N, Hulot C, Lacroix G, Lefèvre JP, Leveque $\mathrm{S}$, Magaud H, Morin A, Pepin G, Pichard A. 2011.Cadmium et ses dérivés. Fiche de données toxicologiques et environnementales des substances chimiques, 82p. http://www.ineris.fr/hml.

Bisson M, Vivier S, La Rocca B, Gourland C. 2009. Point sur les Valeurs Toxicologiques de Référence (VTR), Rapport d'étude 17/03/2009, N DRC08-94380-11776C, INERIS, $60 \mathrm{p}$.

Bliefert C, Perraud R. 2008. Chimie de L'environnement : Air, Eau, Sols, Déchet, (2e éd.). De Boeck : Bruxelles ; 369-389.

Bouye TR, Ocho-Anin AAL, Karamoko M, Otchoumou A. 2017. Étude de la 
croissance d'un escargot géant africain comestible : Achatina achatina (Linné, 1758), élevé sur du substrat amendé à la poudre de coquilles d'escargot. J. Appl. Biosci., 109: 10630-10639. DOI: http://dx.doi.org/10.4314/jab.v109i1.7

Czech A, Stachyra K. 2012. Effect of processing treatments (frozen, frying) on contents of minerals in tissues of 'frutti di mare'. International Journal of Food Science and Technology, 48: 238-245. DOI: $10.1111 / \mathrm{j} .1365-2621.2012 .03179 . \mathrm{x}$

Edorh PA, Agonkpahoun E, Gnandi K, Guedenon P, Koumolou L, Amoussou C, Ayedoun AM, Boko M, Gbeassor M, Rihn BH, Creppy EE. 2009. An assessment of the contamination of Achatina achatina by toxic metals in Okpara village (Benin). Int. J. Biol. Chem. Sci., 3(6): 1428-1436. DOI: http://dx.doi.org/10.4314/ijbcs.v3i6.5354 2

Hounkpatin ASY, Edorh AP, Salifou S, Gnandi K, Koumolou L, Agbandji L, Aissi KA, Gouissi M, Boko M. 2012. Assessment of exposure risk to lead and cadmium via fish consumption in the lacusrian village of Ganvié in Benin republic. Journal of Environmental Chemistry and Ecotoxicology, 4(1): 110. DOI: https://doi.org/10.5897/JECE11.063

Kobia J, Emikpe B, Asare D, Asenso T, Yeboah R, Jarikre T, Jagun-Jubril A. 2016. Effects of Different Cooking Methods on Heavy Metals Level in Fresh and Smoked Game Meat. J Food Process Technol., 7: 617. DOI: 10.4172/2157-7110.1000617

Mbétid-Bessane E. 2006. Analyse de la filière des escargots comestibles dans la Région de l'Equateur en République Centrafricaine. Tropicultura, $\mathbf{2 4}$ (2): 115-119.

DOI : www.tropicultura.org/text/v24n2/115.pd $\mathrm{f}$

Miquel MG. 2001. Les effets des métaux lourds sur l'environnement et la santé.
Rapport 261, Office Parlementaire d'Evaluation des Choix Scientifiques et Technologiques, $365 \mathrm{p}$.

Nica VD, Bura M, Gergen L, HărmănescuM, Bordean D-M. 2012. Bioaccumulative and conchological assessment of heavy metals transfer in a soil-plant-snail food chain. Chemistry Central Journal, 6(55): 1-15. DOI: 10.1186/1752-153X-6-55.

Otchoumou A. 2005. Effet de la teneur en calcium d'aliments composés et de la photopériode sur les performances biologiques chez trois espèces d'escargots Achatinidae de Côte d'Ivoire élevées en bâtiment. Thèse de Doctorat d'Etat ES-Sciences Naturelles en Biologie et Ecologie Animales, Université d'Abobo Adjamé, Abidjan, Côte d'Ivoire, $171 \mathrm{p}$.

Ouro-Sama K, Solitoke HD, Gnandi K, Afiademanyo KM, Bowessidjaou EJ. 2014. Évaluation et risques sanitaires de la bioaccumulation de métaux lourds chez des espèces halieutiques du système lagunaire togolais. VertigO-la revue électronique en sciences de l'environnement, 14(2): 1-17. DOI: 10.4000/vertigo. 15093.

Satoh M, Koyama H, Kaji T, Kito H, Tohyama C. 2002. Perspectives on cadmium toxicity research. Tohoku. J. Exp. Med., 196(1): 23-32. DOI: https://doi.org/10.1620/tjem.196.23

Sika Piba NA, Karamoko M, Adou CFD, Otchoumou A, Kouassi KP. 2014. Effet du régime et de la teneur en protéines brutes alimentaires sur le rendement en viande de l'escargot Achatina fulica (Bowdich, 1720). Int. J. Biol. Chem. Sci., 8(5): 2296-2305. DOI: http://dx.doi.org/10.4314/ijbcs.v8i5.31

Sodjinou E, Biaou G, Codjia J-C. 2002. Caractérisation du marché des escargots géants africains (achatines) dans les départements de l'Atlantique et du Littoral au Sud-Bénin. Tropicultura 20(2): $\quad 83-88 . \quad$ DOI: 
http://www.bib.fsagx.ac.be/tropicultura/p df/v20n2.pdf

Tawfik MS. 2013. Impact of different cooking processes on proximate metals composition of fish and shrimp. Journal of Food Technology, 11(4): 95-102, DOI: 10.3923/jftech.2013.95.102.

Viala A, Grimaldi F. 2005. Ecotoxicologie. In Viala A, Botta A., Eléments de toxicologie $(2 \grave{e} E d)$. Tec \& Doc Lavoisier : Paris ; 245-251.

Waykar B, Petare R.2013. Bioaccumulation of heavy metals in freshwater snails Bellamya bengalensis and Lymnea accuminata from malangaon wetland of dhule district (maharashtra) india. The Bioscan., $\quad$ 8(3): $\quad$ 1043-1047. http://www.thebioscan.in/Journal\%20Su pplement/83Sup16\%20WAYKAR\%20B HALCHANDRA

Yehouenou EAP, Boko M, Cornelis AMVG, Hyacinthe A, LALEYE P, Simon A, Bert VH, Kees S, Nico MVS. 2006 b. Organochlorine and organophosphorous pesticide residues in the Ouémé River catchment in the Republic of Bénin. Environnement Int., 32: 616-623. DOI: 10.1016/j.envint.2006.01.007

Yehouenou EAP, Laleye P, Michel B, Cornelis AMVG, Hyacinthe A, Simon
A, Bert VH, Kees S, Nico MVS.2006a. Contamination of fish organochlorine pesticide residues in the Ouémé River catchment in the Republic of Benin. Environnement Int., 32: 594-5999. DOI: 10.1016/j.envint.2006.01.003

Youssao A, Soclo HH, Bonou C, Vianou K, Gbaguidi M, Dovonon L. 2011a. Evaluation de la contamination de la faune ichthyenne dans le complexe lagunaire Nokoué - chenal de Cotonou par le plomb : cas des espèces Sarotherodon melanotheron, Tilapia guineensis et Hemichromisfasciatus (Bénin). Int. J. Biol. Chem. Sci., 5(2): 595-602.

DOI : http://dx.doi.org/10.4314/ijbcs.v5i2.7212 5

Youssao A, Soclo HH, Bonou C, Fayomi B. 2011b. Evaluation de la bioaccumulation du plomb dans les espèces animales marines et identification des sources de contamination métallique par une analyse multiélémentaire en métaux (Al, $\mathrm{Cd}, \mathrm{Cr}, \mathrm{Cu}, \mathrm{Pb}$ ) dans les eaux côtières du Bénin. Int. J. Biol. Chem. Sci., 5(1): 188195.

DOI: http://dx.doi.org/10.4314/ijbcs.v5i1.6809 7 